\title{
ABOUT DIGITAL PROCESSING OF ASTRONOMICAL IMAGES USING HISTOGRAM PROCESSING METHODS
}

\author{
D. Alyoshin, PhD student, NKU named after M. Kozybayev, Petropavlovsk, Republic of Kazakhstan \\ A. Demianenko, Candidate of Technical Sciences, Professor of the NKU named after M. Kozybayev, \\ Petropavlovsk, Republic of Kazakhstan, \\ A. Solodovnik, Candidate of Physical and Mathematical Sciences, Professor of the NKU named after \\ M. Kozybayev, Petropavlovsk, Republic of Kazakhstan
}

\section{DOI: https://doi.org/10.31435/rsglobal_conf/25042021/7524}

\begin{abstract}
Photos of extended objects are crucial for astronomers, as they contain enough detailed information about the celestial bodies that it is quite difficult to extract visually. Most of the information available for analyzing these objects begins with studying them with telescopes or satellites. Unfortunately, the quality of astronomical images is usually very poor compared to other real images, and this is due to the technical and physical features associated with the process of obtaining them. This increases the percentage of noise and makes it more difficult to directly use standard methods on the original image. Images taken from a satellite or telescope are almost always grayscale, but still contain some color information. However, an astronomical image can be obtained through a color filter. Different photodetectors also usually have different sensitivity to different colors (wavelengths). In our paper, we will present a method for processing astronomical images, using histogram processing, which can be successfully used to improve images, and post-processing.

Keywords: image, histogram, processing, function, pixel.
\end{abstract}

Vision is the most advanced of our senses, so it is not surprising that visual images play a crucial role in human perception. However, unlike humans, who are able to perceive electromagnetic radiation only in the visible range, machine image processing covers almost the entire electromagnetic spectrum from gamma radiation to radio waves. Processed images can be generated by sources that are unusual for a person to associate with the observed images. These are, for example, ultrasound images; images obtained in electron microscopy or generated by a computer. Thus, digital image processing covers a wide and diverse range of applications.

An image can be defined as a two-dimensional function $f(x, y)$, where $\mathrm{x}$ and $\mathrm{y}$ are coordinates in space (specifically, on a plane), and whose $\mathrm{f}$ value at any point given by a pair of coordinates $(x, y)$ is called the intensity or gray level of the image at that point. If the values $x, y$, and $\mathrm{f}$ take a finite number of discrete values, then we are talking about a digital image. Digital image processing is the processing of digital images using digital computers (computers). Note that a digital image consists of a finite number of elements, each of which is located in a specific place and takes a certain value. These elements are called image elements or pixels.

In the entire range from image processing to machine vision, there are no clear boundaries, however, it is possible to distinguish between low-, medium-and high-level computerized processes. Low-level processes only deal with primitive operations such as preprocessing to reduce noise, increase contrast, or sharpen images. Low-level processes are characterized by the fact that there are images at the input and output. Image processing at the middle level covers such tasks as segmentation (dividing an image into areas or selecting objects on it), describing objects and compressing them into a form convenient for computer processing, as well as classification (recognition) of individual objects. Medium-level processes are characterized by images only at the input, while the output receives features and attributes extracted from these images (for example, area boundaries, contour lines, distinctive features of specific objects). Finally, high-level processing involves the "comprehension" of a set of recognized objects, as is done in image analysis, and, to the limit, the implementation of cognitive functions that are commonly associated with vision.

The processing of astronomical images has stages of digital preparation. An important aspect is the registration of the image, which can be extremely simple, as in the case when the original image is already presented in digital form. In general, the image registration stage involves some preprocessing, such as scaling. 
Image enhancement is among the simplest and most impressive areas of digital image processing. Essentially, the idea behind image enhancement techniques is to identify poorly discernible details, or simply emphasize the characteristics of interest in the original image. A well-known example of an improvement is to increase the contrast of an image, because the result is "it looks better". It is important to keep in mind that quality improvement is a highly subjective area in image processing.

In image restoration, where the area is also associated with an increase in the visual quality of the image, however, unlike the actual improvement, the criteria for which are subjective, image restoration is objective in the sense that image restoration methods rely on mathematical or probabilistic models of image distortion. In contrast, image enhancement is based on the subjective preferences of human perception, which are related to what exactly is considered a "good" improvement result.

For example, wavelets form the foundation for representing images with multiple degrees of resolution at the same time. Compression, as the name implies, refers to methods of reducing the amount of memory needed to store an image, or narrowing the channel bandwidth required to transmit it. Although the technology of storage devices has been significantly improved over the past decade, the same cannot be said for the bandwidth of communication lines.

Compression, as the name implies, refers to methods of reducing the amount of memory required to store an image, or narrowing the bandwidth required to transmit it. Although the technology of storage devices has been significantly improved over the past decade, the same cannot be said for the bandwidth of communication lines.

Segmentation divides the image into its component parts or objects. In general, automatic segmentation is one of the most difficult tasks of digital image processing. Unnecessarily detailed segmentation leads the process of solving the image processing problem to a complex path, if you need to identify objects individually. On the other hand, insufficiently detailed or erroneous segmentation will almost inevitably lead to errors at the final stage of processing. In general, the more accurate the segmentation, the more likely it is to succeed in recognition.

When applying these methods of image preparation and processing, it involves selecting a set of elements that will not affect the initial representation of image formation. In this connection, several aspects stand out. The first is geometric, where image formation can be viewed as a projection from 3$\mathrm{D}$ to 2-D space. The disappearance of one coordinate leads to a serious loss of information about the geometry of the observed scene. However, our brain perceives the received information as threedimensional. The second aspect determines how "bright" the object is and how the brightness in the image depends on the optical properties of the object and the imaging system. The third question is what happens to an image when we represent it using an array of discrete numbers to be processed on a computer. What interference to the image is caused by the processing processes.

The main goal of the improvement is to process the image in such a way that the result is more suitable from the point of view of a particular application. The word concrete is important here because it establishes from the very beginning that the methods discussed in this chapter are largely problem-oriented. For example, a method that is very useful for improving X-ray images will not necessarily be the best for processing images of Mars transmitted by a spacecraft. However, regardless of the methods used, image enhancement is one of the most interesting and attractive areas of image processing from the point of view of visual analysis.

Many approaches to image enhancement fall into two broad categories:

- methods of processing in the spatial domain (spatial methods)

- methods of processing in the frequency domain (frequency methods).

There is no general theory of image enhancement. When an image is processed, the visual perception of the results is an indicator of how well a particular method is performing. Visual assessment of image quality is a subjective process. When the goal is to process the image for further processing by the computer, the estimation task is somewhat simpler. For example, in a character recognition problem, the best method (leaving aside other issues such as computational requirements) is the image processing method that gives more accurate recognition results. However, even in a situation where the problem allows you to set clear quality criteria, it usually takes a certain number of testing attempts until a specific approach to image improvement is selected.

Successful experience has shown the use for image processing the application of histogram processing, which can be successfully used to improve images. In addition to getting useful statistics 
about the image, the information contained in the histogram is also very useful in other tasks, such as image compression and segmentation. Histograms are quite simple for both software calculation and hardware implementation, which makes them a convenient tool for real-time image processing.

A digital image histogram with brightness levels in the range $[0, L-1]$ is called a discrete function $h\left(r_{k}\right)=n_{k}$, where $\mathrm{r}_{\mathrm{k}}$ is the $\mathrm{k}$-th brightness level, and $\mathrm{r}_{\mathrm{k}}$ is the number of pixels in the image that have the brightness of $r_{k}$.

A common practice is to normalize a histogram by dividing each of its values by the total number of pixels in the image, denoted by $\mathrm{n}$. Thus, the values of the normalized histogram will be $p\left(r_{k}\right)=n_{k} / n$ for $k=0,1, \ldots, L-1$. Generally speaking, $\mathrm{p}$ is an estimate of the probability of a pixel appearing with a brightness value of $\mathrm{r}_{\mathrm{k}}$. Note that the sum of all the values of the normalized histogram is equal to one.

However, note that the methods of histogram processing were global, where the construction of the transformation function is based on the analysis of the brightness content of the entire image. Although this global approach is suitable for improvement in general, there are cases where it is necessary to improve the details by analyzing small areas of the image. This is due to the fact that the number of pixels in such areas is small and cannot have a noticeable effect on the global histogram, the shape of which does not necessarily correspond to the necessary local improvement.

The solution is to develop a conversion function based on the distribution of brightness (or other characteristics) in the neighborhood of each image element. The methods of histogram processing described earlier can be easily applied to local improvement. The procedure consists in setting the shape of a square or rectangular neighborhood around the element to be processed and then moving the center of this area from the sharpener to the point. For each new position of the neighborhood, a histogram is calculated from the points included in it, and the function of converting the equalization or reducing the histogram is found. Finally, this function is used to display the brightness level of the central element of the neighborhood.

Then the center of the neighborhood is moved to the neighboring pixel and the procedure is repeated. Since only one column or row of the neighborhood changes as you move from point to point, it becomes possible to update the histogram obtained in the previous step by adding new data. This approach has obvious advantages over calculating the histogram again for all points in the neighborhood, with its offset by only one element. Another approach, sometimes used to reduce the number of calculations, is to use disjoint areas, but this method usually leads to an undesirable chessboard effect.

Histograms have many applications in image processing. The first use, as also discussed above, is image analysis. We can predict an image just by looking at its histogram. It's like looking at an X-ray of a body bone.

The second use of the histogram is for brightness purposes. Histograms have a wide application in image brightness. Not only by brightness, but also by histograms is used to adjust the contrast of the image.

Another important use of the histogram is to align the image.

Last but not least, the histogram has a wide application in threshold values. This is mainly used in computer vision.

\section{REFERENCES}

1. Burke B., Gregory J., Cooper M., Loomis A., Young D., Lind T., Doherty P., Daniels P., Landers D., Ciampi J., Johnson K., O’Brien P. CCD Imager Development for Astronomy. Lincoln Laboratory Journal, 2007, Volume 16, Number 2.

2. Gonzalez R. Digital image processing / Gonzalez R., Woods R., - M.: Technosphere, 2005. - 1072 p. ISBN 5-94836-028-8-2000 copies.

3. Gruzman I. S. Digital image processing in information systems: textbook. Manual / I. S. Gruzman, V. S. Kirichuk, V. P. Kosykh, G. I. Peretyagin, A. A. Spektor.- Novosibisrk: NSTU Publishing House, 2002. - 352 p. - 2000 copies. - ISBN 5-7782-0330-6.

4. Yane. B. Digital image processing / Yane. B.-Technosphere, 2007. - 584c. ISBN 978-5-94836-122-2.

5. Wiley K., Connolly A., Gardner J., Krughof S., Balazinska M., Howe B., Kwon Y., Bu Y. Astronomy in the Cloud: Using Map Reduce for Image Coaddition. Publications of the Astronomical Society of the Pacific, 2011, Vol. 123, No. 901, pp. 366-380. 